\title{
Study of the low-temperature line broadening mechanisms for excitonic marsitions in GaAs/AIGaAs modulator structures
}

\author{
S. Hong and Jasprit Singh \\ The University of Michigan, Department of Electrical Engineering and Computer Science, Ann Arbor, \\ Michigan 48109
}

(Received 3 April 1986; accepted for publication 13 June 1986)

\begin{abstract}
We examine the dominant line broadening mechanisms for excitonic lines in a quantum well in the presence of strong transverse electric fields. We have calculated the line broadening due to interface roughness effects and lifetime broadening effects. We find that the line broadening effects due to interface roughness increase rapidly as the transverse electric field is increased and for a $100-\AA \mathrm{Al}_{0.3} \mathrm{Ga}_{0.7} \mathrm{As} / \mathrm{GaAs}$ well, increase by a factor of $\sim 2.0$ as the field is increased from 0 to $100 \mathrm{kV} / \mathrm{cm}$. The broadening due to lifetime effects is very sensitive to the band lineup assumed and is much smaller than interface roughness effects even for one monolayer interface roughness.
\end{abstract}

Recent advances in heterostructure technology have led to the conception and realization of a number of novel device structures. ${ }^{1.2}$ These devices utilize the potential of tailoring the electronic structure of materials and thereby altering their optical and transport properties. An important optoelectronic device structure being studied by several groups is the light modulator based on a quantum well in the presence of a transverse electric field. ${ }^{3-5}$ The interest in this device is due to the potential of shifting the excitonic energies in the quantum well by application of an electric field. This allows the possibility of changing the transmission intensity of a signal beam coming in at a fixed wavelength by applying appropriate electric field. Thus, optical modulation can be produced at high speeds. The dept h of the modulation produced depends on the linewidti of the excitonic peak. Clearly if the shift in the excitonic peak is comparable to the linewidth, the optical modulation action will be lost.

In this letter we examine the low-temperature line broadening mechanisms for the quantum well modulator. In the case of no electric field, for the AlGaAs/GaAs quantum well, the excitonic linewidth is controlled by the perfection of the interface at low temperature ${ }^{6,7}$ However, in the presence of a transverse electric field, the electron and hole levels are only quasibound states which have a finite lifetime in the wells. This produces an additional source of line broadening in the excitonic line. In this letter we present results of calculations for both of these mechanisms. The lifetime broadening is very sensitive to the band lineup assumed, but we find that if the interface roughness is one monolayer or more and the lateral extent of the interface islands (discussed below) is comparable to the exciton radius, the line broadening due to interface roughness dominates.

The exciton problem can be solved by considering the following sets of equations:

(i) For the electron $(e)$, hole $(h)$ subband levels:

$$
\begin{gathered}
\mathscr{H}_{e, h}^{0} \psi_{e, h}=\left[-\frac{\hbar^{2}}{2 m_{e, h}} \frac{\partial^{2}}{\partial z_{e, h}^{2}}+e \mathbf{E} \cdot z+V\left(z_{e, h}\right)\right] \psi_{e, h} \\
=E_{e, h} \psi_{e, h}, \\
V\left(z_{e, h}\right)=V_{e, h} \quad z<0 \quad \text { or } \quad \geqslant W_{0}=0
\end{gathered}
$$

(ii) For the exciton energy and wave function:

$$
\begin{gathered}
{\left[\mathscr{H}_{e}^{0}+\mathscr{H}_{h}^{0}-\frac{\hbar^{2}}{2 \mu}\left(\frac{1}{\rho} \frac{\partial}{\partial \rho} \rho \frac{\partial}{\partial \rho}+\frac{1}{\rho^{2}} \frac{\partial^{2}}{\partial \phi^{2}}\right)\right.} \\
\left.-\frac{e^{2}}{\epsilon\left|r_{e}-\gamma_{h}\right|}\right] \psi_{\mathrm{ex}}=E_{\mathrm{ex}} \psi_{\mathrm{ex}} .
\end{gathered}
$$

Here, $m_{e}, m_{h}$ are the electron and hole effective masses, $\mu$ the exciton reduced mass, $\mathbf{E}$ the electric field, and $\epsilon$ the dielectric constant (assumed same in the well and barrier).

For narrow quantum wells $\left(W_{0}<200 \AA\right)$ the ground state exciton wave function may be assumed to have the form ${ }^{8.9}$

$$
\psi_{\mathrm{ex}}=\psi_{\mathrm{e}} \psi_{h} \exp \left[-\left(\rho^{2}+z^{2}\right)^{1 / 2} / \lambda\right],
$$

where $\lambda$ is determined variationally by energy minimization. The exciton energy is determined by

$$
E_{\mathrm{ex}}=E_{e}+E_{h}+E_{g}-E_{b},
$$

where $E_{\mathrm{g}}$ is the material band gap and $E_{b}$ is the exciton binding energy. In the presence of an electric field, the electron and hole wave functions are quasibound and one can determine their tunneling rates out of the well to a good approximation by the WKB approximation.

We have developed the formalism necessary to solve the above equations using a Monte Carlo approach. ${ }^{10-12}$

To calculate the effect of the interface roughness on the exciton line, we first need to calculate the variation of the exciton energy as a function change in the well size by a monolayer. This is straightforward and we find that the dominant source of the change in the exciton energy at all electric fields is due to the changes in the electron and hole subband levels. The exciton binding energy is relatively insensitive to the well size fluctuations.

The source of the interface roughness is expected to be the growth mode under which the epitaxial growth is taking place. This has been discussed in detail in the literature ${ }^{13}$ so we will only briefly describe the model for interface roughness that we will use for our calculations. A nonideal interface can be represented by a plane at $Z=0$ and fluctuations extending a distance $\delta$ ( $=$ one monolayer or more). We also assume that these fluctuations have a lateral extent (controlled by the growth conditions) which have an area $A_{2 \mathrm{D}}$. We assume that the concentration of the flat regions and 
two-dimensional islands projecting out are equal. The actual interface in a real quantum well is, of course, more complicated, but this simple model has been quite successful in understanding excitonic linewidths in the absence of electric field. ${ }^{13}$ We assume that the islands and flat regions are randomly distributed at the interfacial plane. Due to this random distribution, the exciton energy is position dependent (since the exciton wave function has a finite spatial extent), and in general one may write

$$
E_{\text {ex }}(r, W)=E_{e}(r, W)+E_{h}(r, W)-E_{b}(r, W),
$$

where $r$ now denotes the exciton position and $W$ is the well size averaged over the exciton spatial extent. This average well size will be different at different points of the quantum well. If the excitons are created randomly in the quantum well, then the line shape is determined by the probability distribution $P\left(A_{\mathrm{ex}}, c_{a}\right)$ that there are concentration fluctuations $c_{a}$ and $c_{b}$ over the area of the exciton $A_{\mathrm{ex}}$. To first order the probability of well size

$$
W=W_{0}+\delta\left[\left(c_{a}-c_{a}^{0}\right)-\left(c_{b}-c_{b}^{0}\right)\right]
$$

is $^{6,14}$

$$
P\left(A_{\mathrm{ex}}, c_{a}\right)=\exp -\left[\frac{A_{\mathrm{ex}}}{A_{2 \mathrm{D}}}\left(c_{a} \ln \frac{c_{a}}{c_{a}^{0}}+c_{b} \ln \frac{c_{b}}{c_{b}^{0}}\right)\right],
$$

where $c_{a}^{0}$ and $c_{b}^{0}$ represent the mean concentrations of the flat regions and islands discussed previously, and $c_{a}$ and $c_{b}$ are the concentration fluctuations produced by the randomness of the interface roughness. If $A_{\mathrm{ex}}>A_{2 \mathrm{D}}$, the probability distribution has a narrow width and one may write

$$
P\left(A_{\mathrm{ex}}, c_{a}\right)=\exp -\left(\frac{A_{\mathrm{ex}}}{A_{2 \mathrm{D}}} \frac{\left(c_{a}-c_{a}^{0}\right)^{2}}{2 c_{a}^{0} c_{b}^{0}}\right),
$$

the exciton linewidth is given by

$$
\begin{aligned}
\sigma & =\left[1.4 c_{a}^{0} c_{b}^{0} A_{2 \mathrm{D}} / A_{\mathrm{ex}}\right]^{1 / 2}\left[c_{a}^{0} \Delta^{+}+c_{b}^{0} \Delta^{-}\right] \\
& \cong\left[1.4 c_{a}^{0} c_{b}^{0} A_{2 \mathrm{D}} / A_{\mathrm{ex}}\right]^{1 / 2} \Delta,
\end{aligned}
$$

where

$$
\Delta^{+}=\left.\frac{\partial E_{\mathrm{ex}}}{\partial W}\right|_{W_{0}+\partial W} \delta ; \quad \Delta^{-}=\left.\frac{\partial E_{\mathrm{ex}}}{\partial W}\right|_{W_{n}-\partial W} \delta .
$$

In general, if $A_{\mathrm{ex}} \sim A_{2 \mathrm{D}}$, one determines the full width $\Delta P$ of the distribution $P\left(A_{\mathrm{ex}}, c_{a}\right)$ and the linewidth is then $\sigma=\Delta P \Delta$. Thus $\Delta$ represents the change in exciton energy when the well size changes by $\delta$.

In Fig. 1 we plot the variation of $\Delta$ when $\delta=$ one monolayer, and the exciton lateral extent $[\lambda$ of $\mathrm{Eq}$. (4)] as a function of electric field for $100 \AA$ and $70 \AA \mathrm{AlGaAs} / \mathrm{GaAs}$ wells. We have assumed a 60:40 band lineup (i.e., band-gap discontinuity across the conduction and valence bands). Note that $A_{\text {ex }}=3 / 2$ by $\lambda^{2}$. It is interesting to note that $\lambda$ increases rapidly with applied electric field. One can understand this effect on the following physical basis. As the electric field is increased, the electron and hole wave functions are pushed towards the interface and become more peaked. This causes their eigenvalues to be more sensitive to fluctuations in the well size. As can be seen from Fig. 1, the value of $\Delta$ increases from $3 \mathrm{meV}$ at no field to $8 \mathrm{meV}$ at $100 \mathrm{kV} \mathrm{cm}^{-1}$ for $W_{0}=100 \AA$ well. The change is relatively smaller for the $70 \AA$ well. The lateral extent of the exciton increases as the

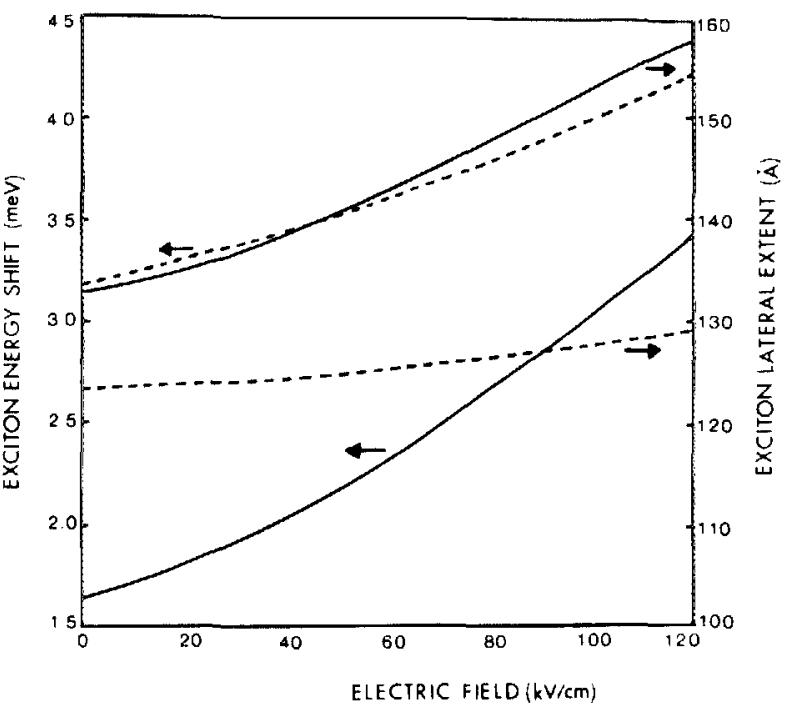

FIG. 1. Change in the exciton energy when the well size changes by a monolayer and the variation of the exciton lateral extent $[\lambda$ of Eq. (4)] as a function of applied transverse electric field. Solid lines-well size $=100 \AA$; dashed lines-well size $=70 \AA$.

electric field is increased. This is because the overlap of the electron and hole wave function decreases as the electric field is increased, thus reducing the exciton binding energy and causing the exciton to spread out. As can be seen from Eqs. (10) and (11), as the electric field is increased, we expect the excitonic linewidth to increase due to the increase in $\Delta$, but to decrease due to the increase in the exciton lateral extent.

The expression for the linewidth derived above is for one interface assuming that the other interface is perfect (a quantum well has two interfaces). However, if the two interfaces are comparable in quality, then the total linewidth in the single quantum well is

$$
\sigma_{\text {tot }}=\sqrt{2} \sigma .
$$

In Fig. 2, we show the exciton linewidth as a function of electric field due to the interface roughness effects and the finite lifetime of the electric field. The results are shown for a $100-\AA \mathrm{Al}_{0.3} \mathrm{Ga}_{0.7} \mathrm{As} / \mathrm{GaAs}$ quantum well and for the interface roughness we show results for $A_{2 \mathrm{D}}=4 \times 10^{2} \AA^{2}$ and $A_{2 \mathrm{D}}=2.2 \times 10^{4} \AA^{2}$ representing two different types of surface roughness. We have assumed $\delta=$ one monolayer (the linewidth increases almost linearly with $\delta$ ). For the lifetime broadening we show results for two different discontinuities for the band lineups for a $100-\AA$ well. Note that the interface roughness limited linewidth is not sensitive to the band lineup value assumed. However, the lifetime broadening is very sensitive to the band lineup assumed. We point out that for a 60:40 discontinuity the lifetime broacening is negligible even at high electric field. However, for the 85:15 discontinuity, the lifetime broadening does become important and can contribute significantly to the linewidth. We point out that it was earlier believed that the band lineup in the GaAs/ AlGaAs system was given by the $85: 15$ lineup,${ }^{15}$ but recent studies show that the lineup is given by the 60:40 distribution. ${ }^{16}$ Thus, it appears that lifetime effects are not significant.

As can be expected from Eq. (10) the interface rough- 


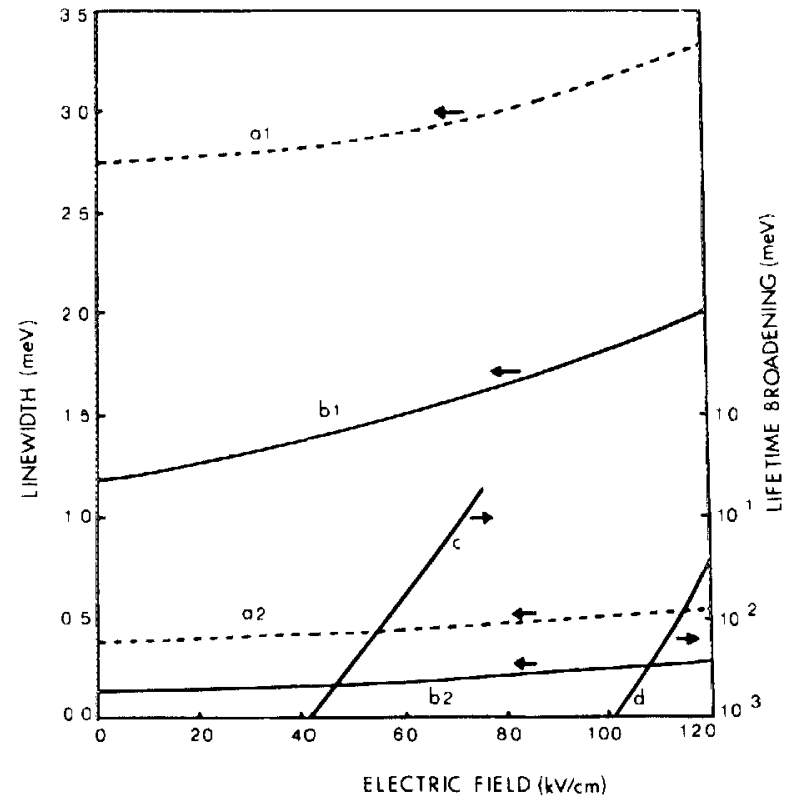

FIG. 2. Excitonic linewid ths due to lifetime broadening and one monolayer interface roughness at each interface of a quantum well as a function of electric field. The different cases are: dashed lines-well size $=70 \AA$; (a1) $A_{2 \mathrm{D}}=2.2 \times 10^{4} \AA^{2}$, (a2) $A_{2 \mathrm{D}}=4.0 \times 10^{2} \AA^{2}$; solid lines-well size $=100$ A; (b1) $A_{2 d}=2.2 \times 10^{4} \AA^{2}$, (b2) $A_{2 \mathrm{D}}=4.0 \times 10^{2} \AA^{2}$; the lifetime results are for (c) 85:15 and (d) 60:40 band lineup.

ness limited linewidth is very sensitive to the nature of the interface roughness (i.e., the two-dimensional size of the islands describing the interface). For a one monolayer height of these islands the linewidth for the case of islands of area $\left(2 \times 10^{4}\right) \AA^{2}$ changes from $1.2 \mathrm{meV}$ at zero field to $2.0 \mathrm{meV}$ at $100 \mathrm{kV} \mathrm{cm}^{-1}$. As can be seen from Fig. 2, the lines are much sharper if the interface is described by islands which are smaller in size.

The above results are for a single quantum well. For most device applications, the optical modulator does not consist of only one quantum well, but is a multiquantum well (MQW) structure. Ideally, of course, the MQW consists of identical single quantum wells, but in practice, there are bound to be small fluctuations in the well size of each quantum well. In this case the excitonic spectra will consist of a series of lines with linewidths calculated above, but with different peak positions superimposed on each other. If the total interwell size fluctuation is $x \delta_{0}$ monolayers (note that $x$ need not be an integer ${ }^{13,17}$ ) the effective width of the spectra will be $\sim x \delta_{0} \Delta$. We point out also that the line shape for narrow wells can be quite asymmetric because $\Delta^{-}=\partial E_{\mathrm{ex}} /\left.\partial W\right|_{\omega_{0}-\partial W} \delta$ is much larger than $\Delta^{+}=\partial E_{\mathrm{ex}} /\left.\partial W\right|_{W_{0}+\partial W^{+}} \delta$ especially for narrow wells. We finally point out that recent experimental measurements of PL linewidths as a function of electric field agree with the predicted increase with the field. ${ }^{18}$

In summary, we have studied the effect of interface roughness and lifetime effects on excitonic linewidths in the presence of high transverse electric field. We find that in realistic device structures, the interface roughness is expected to be the dominant broadening mechanism at low temperatures. The linewidth increases rapidly with the applied electric field and thus interface roughness can cause serious effects on the performance of light modulators.

'E. E. Mendez, G. Bastard, L. L. Chang, L. Esaki, H. Morkoç, and R. Fischer, Phys. Rev. B 26, 7101 (1982).

${ }^{2}$ W. D. Goodhue, B. E. Burke, K. B. Nichols, G. M. Metze, and G. D. Johnson, presented at the 6th MBE Workshop, Aug. 14-16, 1985, Minneapolis, $\mathrm{MN}$.

${ }^{3}$ T. H. Wood, C. A. Burrus, B. A. B. Miller, D. S. Chemla, T. C. Dammen, A. C. Gossard, and W. Wiegmann, Appl. Phys. Lett. 44, 16 (1984).

${ }^{4}$ T. C. Damen, A. C. Gossard, W. Wiegmann, T. H. Wood, and C. A. Burrus, Phys. Rev. B 32, 1043 (1985)

5J. A. Kash, E. E. Mendez, and H. Morkoç, Appl. Phys. Lett. 46, 173 (1985).

${ }^{\circ}$ C. Weisbuch, R. Dingle, P. M. Petroff, A. C. Gossard, and W. Wiegmann, Appl. Phys. Lett. 38, 840 (1981).

'J. Singh and K. K. Bajaj, J. Appl. Phys. 57, 5433 (1986)

${ }^{8} \mathrm{G}$. Bastard, E. E. Mendez, L. L. Chang, and L. Esaki, Phys. Rev. B 26, 1974 (1982).

9R. L. Green, K. K. Bajaj, and D. E. Phelps, Phys. Rev. B 29, 1807 (1984). ${ }^{10}$ J. Singh, Appl. Phys. Lett. 48, 434 (1986).

'J. Singh, J. Appl. Phys. 59, 2953 (1986).

${ }^{12} \mathrm{~J}$. Singh and S. Hong, IEEE J. Quantum Electron. (to be published).

${ }^{13}$ J. Singh, K. K. Bajaj, D. C. Reynolds, C. W. Litton, P. W. Yu, W. T. Masselink, R. Fischer, and H. Morkoç, J. Vac. Sci. Technol. B 3, 1061 (1985).

${ }^{14}$ I. M. Lifshitz, Adv. Phys. 13, 483 (1965).

${ }^{15}$ R. Dingle, in Festkorper-probleme (Advances in Solid State Physics), edited by H. J. Queisser (Pergamon, Braunschweig, 1975), Vol. XV, p. 21.

${ }^{16}$ R. C. Miller, D. A. Klienman, and A. C. Gossard, Phys. Rev. B 29, 7085 (1984)

${ }^{17}$ K. K. Bajaj, D. C. Reynolds, C. W. Litton, J. Singh, P. W. Yu, W. T. Masselink, R. Fischer, and H. Morkoç, Solid State Electron. 29, 215 (1986).

${ }^{18}$ F. Y. Juang, J. Singh, P. K. Bhattacharya, K. Bajema, and R. Merlin, Appl. Phys. Lett. (to be published). 